\title{
Diffusion Weighted Magnetic Resonance Imaging Features of Intracranial Lesions
}

\author{
P. Pradeep Kumar Reddy ${ }^{1}$, P. Madhu² \\ ${ }^{1}$ Senior Resident ${ }^{2}$ Assistant Professor, Department of Radiodiagnosis, Government Medical College, Nizamabad, Telangana. \\ India.
}

Corresponding author: Dr. P. Madhu, MD(Radiodiagnosis), Assistant Professor, Department of Radiodiagnosis, Government Medical College, Nizamabad, Telangana. India.

DOI: 10.21276/ijcmsr.2018.3.2.7

How to cite this article: P. Pradeep Kumar Reddy, P. Madhu. Diffusion weighted magnetic resonance imaging features of intracranial lesions. International Journal of Contemporary Medicine Surgery and Radiology. 2018;3(2):B24-B30.

\section{A B S T R A C T}

Introduction: DWI (Diffusion Weighted Magnetic Resonance Imaging) has a wide range of applications in the evaluation of intracranial pathological conditions. Study was done to describe the features of intracranial lesions on DWI and to compare these features with ADC and T2 FLAIR images.

Material and methods: DW MRI Study was undertaken in 110 patients admitted with suspected intracranial lesions. All the MRI scans in this study were performed using $1.5 \mathrm{~T}$ MRI scanner.

Results: $41(37.3 \%)$ females and, 69 (62.7\%) males were present in the study. The age of the patients ranged from 6 years to 80 years. Infarcts, infective conditions, extra axial mass lesions, intra axial mass lesions, and other conditions like demyelination, posterior reversible encephalopathy syndrome were included in this study. Infarcts comprised the majority of lesions at 56 cases (50.9\% of the total cases studied). Of these acute infarcts constituted 34 cases (60.7\%); 19 (33.9\%) were chronic infarcts and 3 (5.3\%) were subacute infarcts. All cases of acute infarcts and $66.7 \%$ of subacute infarcts showed diffusion restriction. Among intra axial tumors true restriction was noted in 7 cases. $75 \%$ of glioblastoma multiforme showed true diffusion restriction. $100 \%$ of medulloblastomas and $100 \%$ of lymphomas showed diffusion restriction.

Conclusion: Diffusion weighted MRI has been proven to be of excellent use in the characterization of infarcts and in the detection of acute infarcts

Keywords: MRI, Features of Intracranial Lesions

\section{INTRODUCTION}

Diffusion is a term used to describe the movement of molecules in the extra - cellular space due to random thermal motion. This motion is restricted by boundaries such as membranes and macromolecules. Sometimes restrictions in diffusion are directional, depending on the structure of the tissues, and diffusion is also restricted in pathology. The net displacement of molecules diffusing across an area of tissue per second is called the apparent diffusion coefficient (ADC). In areas of restricted diffusion the ADC is low, whereas in areas of free diffusion it is high. A sequence can be sensitized to this motion by applying two gradients on either side of $180^{\circ} \mathrm{RF}$ pulse. In diffusion imaging, normal tissue that exhibits a high ADC has lower signal intensity than abnormal tissue that has a low ADC as the molecules within it are free to move, while diffusion becomes restricted when pathology is present. ${ }^{1}$

Water diffusivity in the extracellular space is inversely related to the constituents of intracellular space; cells with a high nucleus to cytoplasm ratio and tissues with high cellularity cause increased volume of intracellular space, resulting in diminished mobility of water protons with restriction of diffusion. Thus reduced diffusion can be seen in highly cellular tumors such as lymphoma, meningioma and glioblastoma. Several reports have reported an inverse correlation between ADC value and glioma grade for grade II through IV astrocytomas. ${ }^{2}$ Four important clinical applications of DWI in brain tumors have been to assess tumor grade and cellularity, postoperative injury, peritumoral edema, and integrity of white matter tracts. The grade of brain tumor is pivotal in the treatment decision and assessment of prognosis. Of the histologic features for glioma grading, cellularity has been the target of quantitative assessment with DWI.

ADC values cannot be used in individual cases to differentiate glioma types reliably (the ADCs of patients with grade II astrocytoma and glioblastoma overlap). The ADC values of solid gliomas, metastases, and meningioma were in the same range. In cases of lymphomas, however there was a good contras with white matter, with strongly reduced ADC values. Enhancing lesions of the brain include abscesses and tumors. Diffusion-weighted (DW) imaging is more sensitive than conventional MR imaging in distinguishing brain abscesses and cystic tumors. Pus in brain abscesses is 
strongly hyperintense on trace DW images and has a reduced apparent diffusion coefficient (ADC). On the contrary, most necrotic or cystic brain tumors have intermediate signal intensity on DW images and elevated ADC values.

An important clinical application for DWI in demyelinating disease is in establishing a differential diagnosis with other pathologies. There is no established diffusion pattern for demyelinating plaques, but variable ADC patterns in a single MRI study (representing different stages of lesion progression) and rapid changes in such patterns on previous reports are highly suggestive of demyelinating disease. The most common ADC finding is homogeneously facilitated because of vasogenic edema. However, homogeneous restriction, peripheral restriction with a partial or complete dark ring surrounding a bright center, or central facilitation with an isointense peripheral ring or homogeneously isointense can also be seen. ${ }^{3}$

DWI is also an effective way of differentiating an arachnoid cyst from epidermoid tumors. Both lesions present similar signal intensity characteristic of cerebrospinal fluid(CSF) on T1 and T2 sequences. On DWI, epidermoid tumors are hyperintense whereas arachnoid cysts are hypointense, demonstrating high diffusivity. The ADC values of epidermoid tumors are similar to those of the brain parenchyma, whilst ADC values of arachnoid cysts are similar to those of CSF. Thus DWI has a wide range of applications in the evaluation of intracranial pathological conditions. It provides a specific diagnosis in few situations, and adds to the information provided by conventional sequences in many others. It is in this backdrop, that the objectives set out in this research program will enable us to understand the appearances of various intracranial lesions on diffusion weighted images. The signal characteristics of these lesions on ADC images and T2 FLAIR images will also be described.

\section{MATERIAL AND METHODS}

It was a Descriptive study done from September 2014 to August 2016. Patients referred to the Department of Radiodiagnosis, for MRI brain with diffusion weighted imaging. This consists of a study of 110 patients with intracranial lesions detected on imaging. The MRI was done on the advice of the referring doctor and no patient was made to undergo MRI for the sole purpose of this study.

Inclusion criteria: The criteria for inclusion of the patients in the study included those patients who were clinically referred for diffusion weighted MRI of the brain and were detected to have any of these Infarction and hypoxic ischemic injury, Infective conditions, Tumors - extra axial and intra axial, Demyelination, Metabolic or toxic insults to the brain and Degenerative disorders

Exclusion criteria: Patients who are detected to have intracranial bleed were excluded from the study, contraindication to MRI study such as patients with pacemakers, metallic implants and aneurysmal clips

Patients referred for diffusion weighted MRI of the brain, underwent the examination after contraindications for MRI were excluded and consent was taken.

All the MRI scans in this study were performed using $1.5 \mathrm{~T}$
MRI scanner (Siemens avanto 18 channel)

\section{MRI Protocol}

MRI protocol consisted of the following $-A$ head coil was used $\bullet$ Axial diffusion weighted images of the brain $\bullet$ Sagittal T1W images of the brain - Axial T2W FLAIR images of the brain $-\mathrm{ADC}$ images were reconstructed from the diffusion weighted images $B$ value of 0 and $1000 \mathrm{~s} / \mathrm{mm}^{2}$ were used for diffusion weighting.

\section{RESULTS}

The present study was carried out to describe imaging characteristics of intracranial lesions on DWI and to compare them with ADC and T2 FLAIR images. In 110 cases of intracranial lesions were compiled and analyzed. Of the 110 patients studied, $69(62.7 \%)$ were males and 41 (37.3\%) were females.

The age of the patients with intra cranial lesions studied

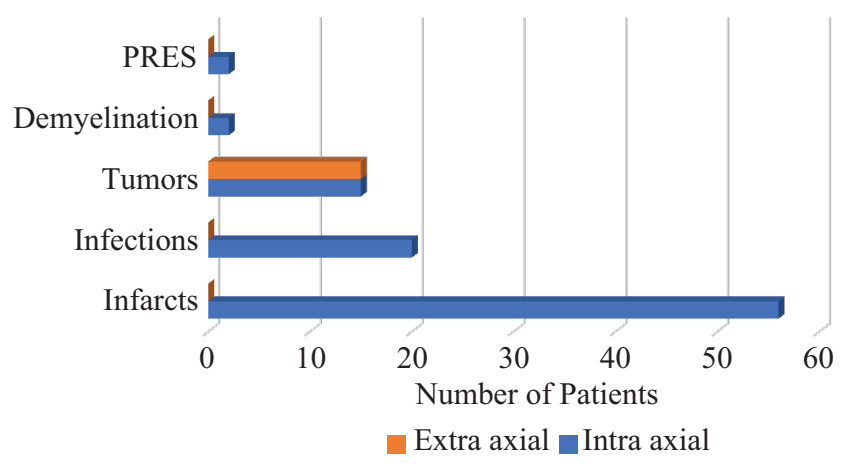

Figure-1: Spectrum of intracranial lesions.

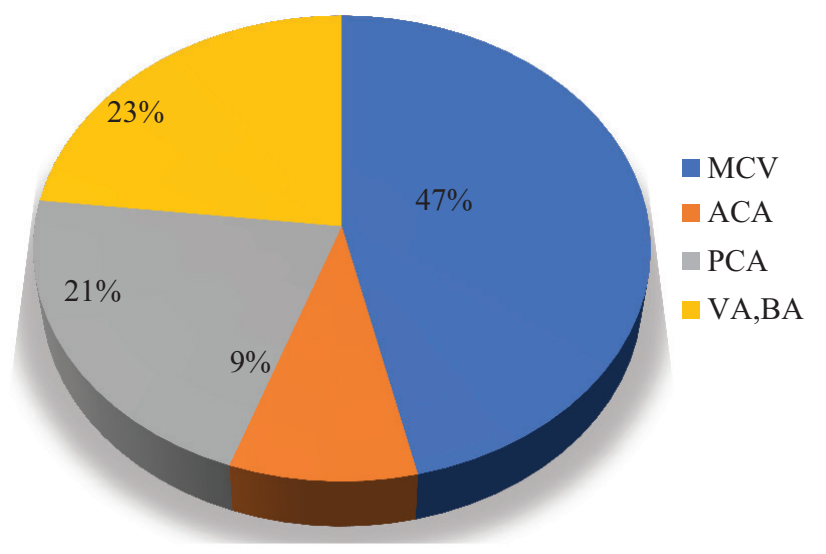

Figure-2: Distribution of Infarcts

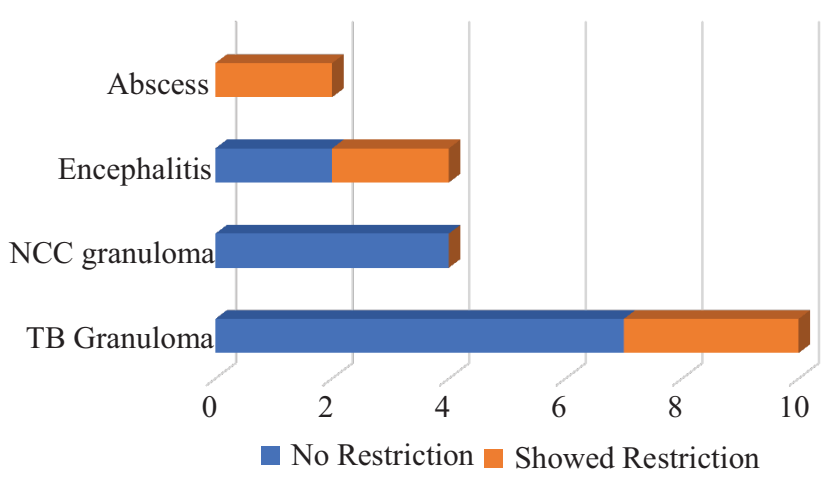

Figure-3: Infective conditions 


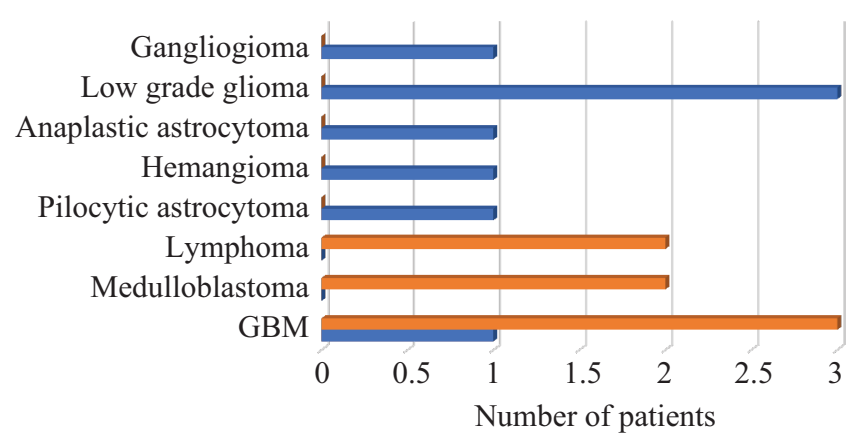

Figure-4: Intra axial tumors

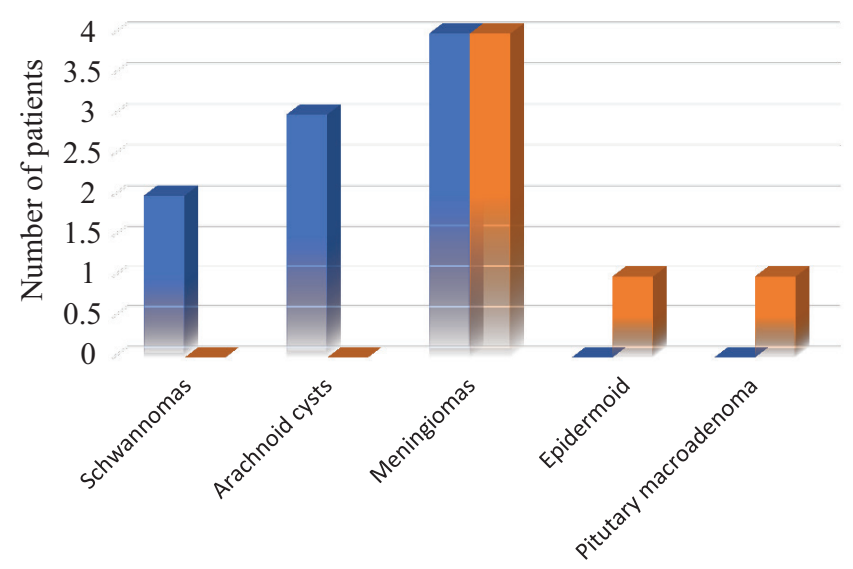

$\square$ No Restriction $\square$ Showed Restriction

Figure-5: Extra axial tumors

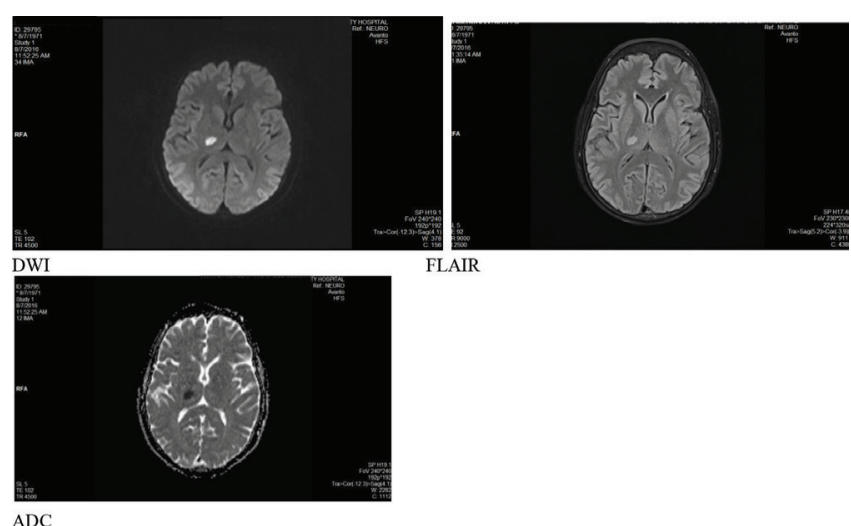

Figure-6: MRI images of a Case - Acute infarct in right thalamus

ranged from 6 years to 80 years. The patients involved in the study were divided into 7 age groups viz. 1-10 years, 11-20 years, $21-30$ years, $31-40$ years, $41-50$ years, $51-60$ years and 61-70 years. There were three patients $(2.7 \%)$ in $1-10$ year age group, nine patients (8.1\%) in 11-20 year age group, fourteen patients (12.7\%) in 21-30 year age group, fourteen (12.7\%) in 31-40 year age group, seventeen (15.5\%) in 41-50 year age group, twenty six (23.6\%) in 51-60 year age group, twenty one (19.1\%) in 61-70 year age group, six (5.4\%) in 71- 80 year age group as given in the table-1.

Intra cranial lesions were found in patients of all age groups. However the peak (23.6\%) was noted in 51-60 year age group Of the total cases included in this study, infarcts were the majority which constituted 56 cases (50.9\%). The other cases

\begin{tabular}{|c|c|c|c|c|c|c|c|c|c|}
\hline \multirow{2}{*}{$\begin{array}{l}\text { Intra } \quad \text { cranial } \\
\text { lesion }\end{array}$} & \multicolumn{8}{|c|}{ Age Range } & \multirow[t]{2}{*}{ Total } \\
\hline & 1-10 & $11-20$ & $21-30$ & $31-40$ & $41-50$ & $51-60$ & $61-70$ & $71-80$ & \\
\hline Acute Infarct & & & 3 & 5 & 4 & 8 & 12 & 2 & 34 \\
\hline Chronic Infarct & & & & & 1 & 8 & 7 & 3 & 19 \\
\hline Subacute Infarct & & & & 1 & & 1 & 1 & & 3 \\
\hline TB granuloma & & 2 & 3 & 1 & 4 & & & & 10 \\
\hline NCC granuloma & & 2 & 1 & & & 1 & & & 4 \\
\hline Encephalitis & 1 & 1 & & 1 & & 1 & & & 4 \\
\hline Meningiomas & & & 1 & 1 & 3 & 2 & 1 & & 8 \\
\hline Schwannomas & & & & 1 & & 1 & & & 2 \\
\hline $\begin{array}{l}\text { Pitutary macro } \\
\text { adenoma }\end{array}$ & & & 1 & & & & & & 1 \\
\hline Epidermoid cyst & & & 1 & & & & & & 1 \\
\hline Arachnoid cyst & 1 & & & 1 & 1 & & & & 3 \\
\hline GBM & & & & & & 3 & & 1 & 4 \\
\hline Low grade glioma & & & & & 2 & 1 & & & 3 \\
\hline Medulloblastoma & & 2 & & & & & & & 2 \\
\hline Lymphomas & & & 1 & 1 & & & & & 2 \\
\hline Pilocytic astrocytoma & 1 & & & & & & & & 1 \\
\hline Hemangioblastoma & & & & & 1 & & & & 1 \\
\hline Ganglioma & & & 1 & & & & & & 1 \\
\hline $\begin{array}{l}\text { Anaplastic } \\
\text { Astrocytoma }\end{array}$ & & & & 1 & & & & & 1 \\
\hline Abscess & & 1 & 1 & & & & & & 2 \\
\hline PRES & & 1 & 1 & & & & & & 2 \\
\hline Multiple Sclerosis & & & & 1 & 1 & & & & 2 \\
\hline TOTAL & 3 & 9 & 14 & 14 & 17 & 26 & 21 & 6 & 110 \\
\hline
\end{tabular}

Table-1: Age wise distribution of intracranial lesions.

were 30 cases of tumors (27.3\%) of which 15 (50\%) were intra axial and 15 (50\%) were extra axial tumors, 20 infective conditions (18.2\%), 2 cases of demyelination (1.8\%) and 2 posterior reversible encephalopathy syndrome $(1.8 \%)$ cases (figure-1).

Of the 110 patients included in this study, 76 cases (69.1\%) showed hyperintensity on DWI of which true restriction (hyperintense on DWI and hypointense on ADC) was noted in 56 patients $(50.9 \%)$. This constituted $73.6 \%$ of the cases showing diffusion restriction. T2 shine through was noted in 17 patients (15.4\%). This constituted $22.4 \%$ of the cases showing diffusion restriction. Three cases were hyperintense on DWI and isointense on ADC. This constituted 3.9\% of the cases showing restriction (figure-2).

56 cases (50.9\%) showed hypointensity on ADC images. All of these were hyperintense on DW images. 19 patients (17.3\%) showed T2 washout (hyperintense on T2WI and isointense on DWI). 2 patients (1.8\%) showed no signal change on DWI or ADC images. 49 patients (44.5\%) had lesions that showed increased diffusivity (hyperintense signal on ADC image). Of these 13 (11.8\%) were hypointense on DWI. This constituted $26.5 \%$ of the cases showing increased diffusivity. 19 of these showed T2 washout, and 17 showed T2 shine through.

\section{Infarcts}

Infarcts constituted 56 cases $(50.9 \%)$ of the total cases in this study. Of this 34 (60.7\%) were acute infarcts, 19 (33.9\%) were chronic infarcts and $3(5.3 \%)$ were subacute infarcts. The age group of patients with infarcts ranged from 24years to 80 years. There were 35 (62.5\%) males and $21(37.5 \%)$ females among these cases.

In 5 cases (8.9\%) the infarcts were in ACA territory, in 26 cases (46.4\%) they were in MCA territory, in 12 cases (21.4\%) the infarcts were in PCA territory and in 13 cases (23.2\%) they were in basilar artery and vertebral artery territory. All 34 cases (100\%) of acute infarcts showed true diffusion restriction with hyperintensity on DWI and hypointensity on ADC images. Of these, 30 cases (88.2\%) showed hyperintensity on T2W images. The remaining 4 
cases $(11.8 \%)$ showed no signal change on $\mathrm{T} 2 \mathrm{~W}$ images. Of the 19 cases of chronic infarcts, ADC signal was increased in all, suggesting increased water diffusivity. In 9 cases (47.3\%), there was hypointensity on DWI and T2 FLAIR images with hyperintensity on ADC images indicating encephalomalacic changes. T2 shine through was noted in 10 cases (52.7\%). None of the cases showed T2 washout.

Out of 3 cases of subacute infarcts, 2 (66.7\%) showed true restriction and $1(33.3 \%)$ showed $\mathrm{T} 2$ shine through.

The study included 20 infective conditions of which 10 (50\%) were tubercular granulomas, 4 (20\%) were NCC granulomas, 2 (10\%) were abscesses, 4 (20\%) were encephalitis (figure-3). True restriction of diffusion was noted in $7(35 \%)$ cases. This included 3 tubercular granulomas 2 abscesses and 2 encephalitis. Thus $30 \%$ of tubercular granulomas, $100 \%$ of abscesses and $50 \%$ of encephalitis showed true diffusion restriction.

T2 washout was seen in all 4 cases (100\%) of NCC granulomas and 6 cases $(60 \%)$ of tubercular granulomas and 1 case of encephalitis (25\%). T2 shine through was seen in 1 case of tubercular granuloma(10\%) and one case of Encephalitis (25\%).

\section{Intra axial tumors}

There were 15 cases of intra axial tumors in this study. The age of the patients ranged from 8 years to 75 years with 9 males and 6 females. This included 4 cases of GBM, 3 cases of Low grade glioma, 2 cases of medulloblastomas, 2 cases of lymphomas 1 case of anaplastic astrocytoma, 1 case of hemangioblastoma, 1 case of pilocytic astrocytoma and 1 case of ganglioglioma (figure-4).

7 cases (46.7\%) showed true diffusion restriction. Of these were 3 were GBM, 2 were medulloblastomas, and two were lymphomas. Thus $75 \%$ of GBM, $100 \%$ of medulloblastomas, and $100 \%$ of lymphomas showed true restriction of diffusion. T2 shine through was noted in 3 cases (20\%). This included 1 case of anaplastic astrocytoma, 1 case (25\%) of GBM, 1 case (33.3\%) of low grade gliomas

T2 washout was seen in 5 cases of which 2 cases were of low grade gliomas, 1 was pilocytic astrocytoma, 1 was ganglioglioma, 1 was hemangioblastoma

15 cases of extra axial tumors were included in this study with an age range of 6 to 63years. Of these 7 were males and 8 were females. These were 8 cases of meningiomas, 3 cases of arachnoid cysts, 2 cases of schwannomas, 1 epidermoid cyst, 1 case of pituitary macroadenoma (figure-5).

True restricted diffusion was noted in 6 cases (40\%). This included the single case of epidermoid cyst, 1 case of pituitary macroadenoma and 4 cases (50\%) of meningiomas. T2 shine through was noted in one case of meningioma. In 3 (37.5\%) cases of meningiomas, T2 FLAIR showed hypointense signal probably due to high cellularity and presence of calcification. 1 case (50\%) of schwannoma showed T2 washout.

Two cases (1.8\%) of demyelination were noted in this study. Both were multiple sclerosis, Both the lesions showed hyperintensity on T2 FLAIR images. True restriction of diffusion was not noted in any of the cases. T2 washout was seen in one case $(50 \%)$. No change was noted on DWI or ADC images in one case (50\%) of multiple sclerosis. Posterior reversible encephalopathy syndrome Two cases $(1.8 \%)$ of
PRES were included in this study. Both the lesions were hyperintense on T2 FLAIR images. One case showed T2 washout (hyperintense on ADC and isointense on DWI). One case showed no change on DWI or ADC images. There was no diffusion restriction in both the cases (figure-6).

\section{DISCUSSION}

Diffusion-weighted (DW) magnetic resonance (MR) imaging provides potentially unique information on the viability of brain tissue. It provides image contrast that is dependent on the molecular motion of water, which may be substantially altered by disease. It is particularly sensitive for detection of acute ischemic stroke and differentiation of acute stroke from other processes that manifest with sudden neurologic deficits. Diffusion weighted MR imaging also provides adjunctive information for other cerebral diseases including neoplasms, intracranial infections, traumatic brain injury, and demyelinating processes. DWI is performed with a pulse sequence capable of measuring water translation over short distances. This water diffusion is much slower in certain pathological conditions as compared with normal brain. ${ }^{4}$

In this study 110 patients with intracranial lesions detected on DW MRI of the brain were included. It was found that DW MRI provides adjunctive information for intracranial lesions including stroke, neoplasms, infections, hypoxic ischemic encephalopathy and extra axial lesions in conjunction with conventional MRI.

\section{Acute infarcts}

In the present study $100 \%$ of acute infarcts showed true restriction. In 4 cases that is $11.7 \%$ of the cases showed no changes in T2WI and FLAIR images. Thus DWI is superior to conventional MR images in detecting acute infarct. Pamela ws et al concluded that DW images are very sensitive and specific for the detection of hyperacute and acute infarctions, with a sensitivity of $88 \%-100 \%$ and a specificity of $86 \%-100 \%$. A lesion with decreased diffusion is strongly correlated with irreversible infarction. ${ }^{5}$

The sensitivity and specificity of DWI in the detection of acute ischemia is $100 \%$. The difference in sensitivity of DWI and conventional MRI sequences is more in the initial time period and decreases as time progresses. Results of this study are correlated with a study done by Gonzalez et al who concluded that DWI is superior to conventional MRI in the diagnosis and characterization of acute infarct. ${ }^{6}$

Study by Rima K et al showed that restricted diffusion is present in all patients on DWMR studies obtained within 24 hours of the onset of symptoms, and in $94 \%$ of patients scanned after 2 weeks after ictus. ${ }^{4}$ P.A.Barber et al study showed sixteen of 17 patients had a final diagnosis of stroke. Acute ischemic changes were seen in all 16 on DWI (100\% sensitivity) and in 12 of 16 on CT. ${ }^{7}$

\section{Subacute and chronic infarcts}

In the present study out of 3 subacute infarcts 2 cases that is $66.7 \%$ of the cases showed restricted diffusion. One case(33.3\%) of subacute infarct showed T2 shine through.19 cases were of chronic infarcts 9 cases(47.3\%) showed hypointensity both on DWI and FLAIR suggestive of encephalomalacia changes.10 cases(52.7\%) of chronic 
infarcts showed T2 shine through that is hyper intensity on both ADC and DWI. Lansberg MG et al study showed the lesion ADC gradually increased from low values during the first week to pseudonormal during the second week to supranormal thereafter. A low ADC value indicated, with good sensitivity (88\%) and specificity (90\%), that a lesion was less than 10 days old. Signal intensity on DWI showed no significant change during the first week but decreased thereafter. Signal intensity on T2 initially increased, decreased slightly during week 2 , and again increased after 14 days. Signal intensity on FLAIR showed the same initial increase as the Signal intensity T2 but remained relatively stable thereafter. ${ }^{8}$

Lutsep HL et al study concluded that Of 103 scans obtained a mean of 10.4 days after symptom onset, DWI detected six lesions not seen on T2WI and discriminated two new infarcts from old lesions. ADC values were low at less than 1 week after stroke onset and became elevated at chronic time points. T2 ratios were near normal acutely, increasing thereafter. ${ }^{9}$

In this study $46.4 \%$ of the infarcts were noted in MCA territory, 9\% were noted involving ACA territory, $21.4 \%$ were noted involving PCA territory and $23.2 \%$ of infarcts were noted involving veterbral and basilar artery territory. This is comparable to a study done by Van Der Zwan et al which showed that MCA territory is the most common site for infarcts and ACA territory is the least common among major arterial territories. ${ }^{10}$

\section{Tumors Extra axial tumors}

In the present study out of 15 extra axial tumors 8 were meningiomas. Out of 8 cases 4 were showing restricted diffusion. Sherif A. Khedr et al study concluded that atypical and malignant meningiomas tend to be markedly hyperintense on diffusion-weighted MR images and exhibit marked decrease in the ADC values when compared with normal brain parenchyma. Although benign meningiomas have a variable appearance on diffusion-weighted images, they tend to have high ADC values compared with normal brain, with the exception of densely calcified or psammomatous meningiomas, which may have a low ADC value. Benign meningiomas with the highest $\mathrm{ADC}$ value seem to have increased water content because of either a specific histological subtype of meningioma or distinct histopathological features. Furthermore, the average ADC values of malignant and atypical meningiomas were significantly lower compared with benign meningiomas. ${ }^{11}$ Yamasaki et al study found lower ADC values in atypical meningiomas than in typical meningiomas, with no significant difference. ${ }^{12}$ Filippi et al reported that atypical (WHO grade 2) and anaplastic (WHO grade 3) meningiomas exhibited lower ADC than that of benign meningioma subtypes. ${ }^{13}$

In the present study all 3 arachnoid cysts showed signal intensity similar to CSF on DWI and ADC. Single case of epidermoid cyst showed restricted diffusion. Hakyemez B et al study concluded that arachnoid cysts had the same intensity with cerebral spinal flow in all sequences. Mean ADC value was 3.41 +/- 0.17 x $10(-3) \mathrm{mm} 2 / \mathrm{sn}$. All epidermoid cysts on diffusion-weighted trace images were more hyperintense than brain parenchyma. The mean ADC value of the epidermoid cysts was $1.15+/-10(-3) \mathrm{mm} 2 / \mathrm{sn}$. The ADC values of the epidermoid cysts were lower than the arachnoid cysts $(p<0.001)$, but were higher than the cerebral white matter $(\mathrm{p}<0.01) .{ }^{14}$ Dutt SN et al study concluded that on MRI, epidermoids and arachnoid cysts usually appear hypointense on T1-weighted images and hyperintense on T2-weighted images. On fluid-attenuated inversion recovery, an arachnoid cyst tends to follow cerebrospinal fluid intensity, whereas an epidermoid becomes hyperintense. ${ }^{15}$ The major differential consideration for the epidermoid cyst is an arachnoid cyst. Arachnoid cysts are isointense to CSF at all sequences, including FLAIR. They displace rather than invade structures such as the epidermoid. Finally, arachnoid cysts do not restrict on diffusion- weighted images. ${ }^{16}$ Hence diffusion weighted imaging is helpful in differentiating between arachnoid cyst and epidermoid cyst.

Single case of pituitary macroadenoma was included in the present study. Restricted diffusion was noted in this case. 2 cases of schwannomas were included. Diffusion restriction was not noted in both the cases. One case showed T2 washout that is the lesion was hyperintense on ADC and iso intense on DWI images. R. Nuri Sener et al study included 6 cases of vestibular schwannomas and concluded that the vestibular schwannomas were isointense to the normal brain parenchyma on diffusion-weighted images and the ADC values of the vestibular schwannomas were prominently higher than that of the normal brain parenchyma. ${ }^{17}$

\section{Intra axial tumors}

MR imaging is the most sensitive method of detecting tumors of the brain. It is however not specific enough to determine the histological nature of most tumors. DWI can differentiate between tumor and infection and can provide information about the cellularity of tumors thereby helping in characterization and grading of tumors.

In the present study 15 intra axial tumors were included. 4 cases were of GBM. True restriction was noted in 3 cases $(75 \%)$ of GBM. One case of GBM showed T2 shine through. 3 cases were of low grade glioma.T2 washout was noted in 2 cases of low grade glioma and one case showed T2 shine through. Single case of anaplastic astrocytoma showed T2 shine through. None of the low grade gliomas and anaplastic astrocytoma showed diffusion restriction. T2 washout was noted in both pilocytic astrocytoma and gangioglioma.

Chen $\mathrm{J}$ et al concluded that The mean $\mathrm{ADC}$ value of the high-grade astrocytomas was significantly lower than that of the low-grade astrocytomas. The mean cellularity of the high-grade astrocytomas was significantly higher than that of the low-grade astrocytomas. ADC value of the astrocytoma was significantly and negatively correlated with its cellularity. Magnetic resonance diffusion weighted imaging may well be highly potential in predicting the degree of astrocytoma. ${ }^{18}$

2 cases of medulloblastomas were noted in the present study and both of them showed restricted diffusion. FF Mohamed et al study included 7 cases of medulloblastoma and showed that In medulloblastoma cases, the diffusion was significantly restricted due to characteristic densely packed cells. In their study all of them appeared predominantly hyperintense on DWI relative to the normal brain parenchyma, hypointense 
on ADC map, which is consistent with marked restriction of diffusion. ${ }^{19}$

M Rodallec et al study included 2 cases of medulloblastomas and concluded that the tumors were of high intensity on diffusion-weighted images with low ADC value. Diffusionweighted MR imaging may be useful for the diagnosis of cerebellar medulloblastoma, due to their high cellularity and high nuclear-to-cytoplasmic ratio. ${ }^{20}$

In the present study the single case of hemangioblastoma showed high signal on ADC images in its solid component suggesting high water diffusivity. F. A. Quadery et al included 9 cases oh hemangioblastomas and in nine of the haemangioblatomas, the solid and contrast-enhancing portions gave low signal on DWI. The ADC was increased in haemangioblastomas. These findings may indicate rich vascular spaces of the haemangioblastomas. ${ }^{21}$

\section{Infections}

In the present study 10 tubercular granulomas and 4 NCC granulomas were included. Out of 10 tubercular granulomas $3(30 \%)$ cases showed diffusion restriction. All the 4(100\%) NCC granulomas and 6(60\%) tubercular granulomas showed T2 washout. One (10\%) case of tubercular granuloma showed T2 shine through. Gupta RK et al concluded that Vesicular and degenerating stages of cysticercus cysts from the core showed ADC values which were significantly higher than the core of all groups of tuberculomas and tuberculous abscess. Their study concluded that addition of DWI to routine imaging protocol may help in differentiation of tuberculous lesions from degenerating cysticercus granuloma. ${ }^{22}$

Reddy JS et al concluded that the ADC value of the tumor cysts was significantly lower compared with benign cysts and neurocysticercosis among nonabscess group. The sensitivity of DWI for the differentiation of brain abscesses from nonabscesses was 96\%; specificity, 96\%; positive predictive value, $98 \%$; negative predictive value, $92 \%$; and accuracy of the test, $96 \%{ }^{23}$

In the present study 4 cases of encephalitis were included. 2 cases (50\%) showed restriction. One case showed T2 shine through and other case showed T2 washout. K Tsuchiya et al concluded that diffusion-weighted imaging was superior to other diagnostic imaging sequences (fluid-attenuated inversion-recovery sequences in eight patients and a T2weighted spin-echo sequence in one patient). Diffusionweighted imaging can serve as a method of detecting early encephalitic changes. ${ }^{24}$

Kiroğlu Y et al proved that DWI is superior to other conventional diagnostic MR sequences in the detection of early viral encephalitic lesions and depiction of the lesion borders and, in combination with other sequences, DWI may contribute to the determination of the disease phase..$^{25}$

\section{Demyelination}

Two cases of demyelination were noted in the present study. Both were multiple sclerosis, Both the lesions showed hyperintensity on T2 FLAIR images. True restriction of diffusion was not noted in any of the cases. T2 washout was seen in one case. No change was noted on DWI or ADC images in one case of multiple sclerosis. K Tsuchiya et al study included 94 cases of multiple sclerosis of which a total of 13 of these plaques showed enhancement on contrast-enhanced T1-weighted images and hyperintensity on DWIs, and five non-enhancing plaques showed hyperintensity on DWIs. Diffusion-weighted imaging, which provides information based on pathophysiology different from contrast-enhanced imaging, is a potential supplementary technique for characterizing MS plaques. ${ }^{26}$

\section{Posterior reversible encephalopathy syndrome}

Two cases of PRES were included in the present study. Both the lesions were hyperintense on T2 FLAIR images. One case showed T2 washout. One case showed no change on DWI or ADC images. Chou MC et al study included 12 cases of PRES and diffusion weighted imaging was done in 10 cases. ${ }^{27}$

\section{CONCLUSION}

Diffusion weighted MRI is a valuable technique that provides unique information about the physiological state of brain tissue.

DWI helps in the characterization of tumors and grading of tumors and can provide valuable information about tumor cellularity. True restriction was not observed in low grade gliomas. The solid portion of high grade tumors may show restricted diffusion. DWI also helps in differentiating medulloblastomas from other posterior fossa lesions such as hemangioblastomas and pilocytic astrocytomas as medulloblastomas show restricted diffusion where as the latter does not show diffusion restriction. Lymphomas may also show restricted diffusion due to their high cellularity. DWI plays an important role in the evaluation of extra axial cystic lesions. Among extra axial tumors, restricted diffusion has been noted in meningiomas. There is no restriction of diffusion in schwannomas. DWI helps in differentiating epidermoid cyst and arachnoid cyst as the former shows restricted diffusion while the latter does not.

Abscesses in DWI shows restricted diffusion due to the high viscosity of the coagulative necrosis and thus helps differentiate them from necrotic tumors which do not show restricted diffusion in their center.

\section{REFERENCES}

1. John R. Haaga, Vikram S. Dogra, Michael forsting, Robert C. Gilkeson, Hyun kwon ha, Muralisundaram. $\mathrm{CT}$ and MRI of the whole body. Fifth edition. China: Elsevier; 2009. p.54,220.

2. Scott W. Atlas, ed., Magnetic resonance imaging of the brain and spine, 4 th edition. China. Lippincott Williams and Wilkins. 2009. p. 472-474.

3. Fabiola W. Cartes-Zumelzua, IoannisStavroub, Mauricio Castilloc Edith Eisenhubera, Engelbert Knospband Majda M. Thurnhera. Diffusion-Weighted Imaging in the Assessment of Brain Abscesses Therapy. AJNR. 2004; 25(1): 1310- 1317.

4. K Rima, G Rohit, P Anjali, C Veena. Role of diffusion weighted MR imaging in early diagnosis of cerebral infarction. Ind J RadiolImag 2003;3(2):213-217.

5. Pamela WS, Ellen Grant and Gilberto Gonzalez. Diffusion-weighted MR Imaging of the Brain. Radiology 2000; 217(3):331-345. 
6. Gonzalez RG, Schaefer PW, Buonanno FS, et al. Diffusion-weighted MR imaging: diagnostic accuracy in patients imaged within 6 hours of stroke symptom onset. Radiology 1999;210(5):155-162.

7. P.A. Barber, D.G. Darby, P.M. Desond, R.P. Gerraty, Q.Yang, T.Li, D. Jolley, G.A. Donnan, B.M. Tress and S.M. Davis. Identification of Major Ischemic Change. Diffusion-Weighted Imaging Versus Computed Tomography, stroke.1999;30(6):2059- 2065.

8. Lansberg MG, Thijs VN, O'Brien MW, Ali JO, de Crespigny AJ, Tong DC, Moseley ME, Albers GW. Evolution of apparent diffusion coefficient, diffusionweighted, and T2-weighted signal intensity of acute stroke. AJNR Am J Neuroradiol 2001;22(4):637-44.

9. Lutsep HL, Albers GW, DeCrespigny A, Kamat GN, Marks MP, Moseley ME. Clinical utility of diffusionweighted magnetic resonance imaging in the assessment of ischemic stroke. Ann Neurol. 1997;41(5):574-80.

10. Van der Zwan A, Hillen B, Tulleken $H$ et al. variability of the major cerebral arteries. J Neurosurg 1992;77(3):927940.

11. Sherif A. Khedr, Mohamed A Hassaan, AmalRefaat. The diagnostic value of diffusion weighted imaging in patients with meningioma. The Egyptian Journal of Radiology and Nuclear Medicine 2012;43(2):249-256.

12. Yamasaki F, Kurisu K, Satoh K, Arita K, Sugiyama K, Ohtaki M, Takaba J, Tominaga A, Hanaya R, Yoshioka H, Hama S, Ito Y, Kajiwara Y, Yahara K, Saito T, Thohar MA. Apparent diffusion coefficient of human brain tumors at MR imaging. Radiology 2005;235(5):985-91.

13. Filippi CG, Edgar MA, Ulu AM et al. Appearance of meningiomas on diffusion- weighted images: correlating diffusion constants with histopathologic findings. AJNR AmJ Neuroradiol 2001; 22(6):65-72.

14. Hakyemez B, Yildiz H, Ergin N, Uysal S, Parlak M. Flair and diffusion weighted MR imaging in differentiating epidermoid cysts from arachnoid cysts. TaniGirisimRadyol. 2003;9(4):418-26.

15. Dutt SN, Mirza S, Chavda SV, Irving RM. Radiologic differentiation of intracranial epidermoids from arachnoid cysts. Otol Neurotol. 2002;23(1):84-92.

16. Anne G. Osborn, MD, and Michael T. Preece, MD. Intracranial Cysts: Radiologic- Pathologic Correlation and Imaging Approach. RSNA, 2000: 239: 3(5):650664 .

17. R. Nuri Sener. Diffusion Magnetic Resonance Imaging of Solid Vestibular Schwannomas. Case Report, Journal of Computer Assisted Tomography 27(2):249- 252.

18. Chen J, Xia J, Zhou YC, Xia LM, Zhu WZ, Zou ML, Feng DY, Wang CY. Correlation between magnetic resonance diffusion weighted imaging and cell density in astrocytoma. ZhonghuaZhong Liu ZaZhi. 2005;27(5):309-11.

19. FatenFawzy Mohamed,Ahmed A. Azeem Ismail, Doaa Ibrahim Hasan,Waleed Elhady Essa. The role of apparent diffusion coefficient (ADC) value in the differentiation between the most common pediatric posterior fossa tumors. The Egyptian Society of Radiology and Nuclear Medicine 44(2):349-355.

20. Rodallec M, Colombat M, Krainik A, Kalamaridès M, Redondo A, Feydy A. Diffusion-weighted MR imaging and pathologic findings in adult cerebellar medulloblastoma.J Neuroradiol. 2004;31(3):234-7.

21. Quadrery FA, Okamoto K. Diffusion-weighted MRI of haemangioblastomas and other cerebellar tumours. Neuroradiology 2003;45(4): 212-219.

22. Gupta RK, Prakash M, Mishra AM, Husain M, Prasad KN, Husain N. Role of diffusion weighted imaging in differentiation of intracranial tuberculoma and tuberculous abscess from cysticercus granulomas-a report of more than 100 lesions. Eur J Radiol. 2005;55(3):384-92.

23. Reddy JS, Mishra AM, Behari S, Husain M, Gupta V, Rastogi M, Gupta RK. The role of diffusion-weighted imaging in the differential diagnosis of intracranial cystic mass lesions: a report of 147 lesions. Surg Neurol. 2006;66(3):246-50.

24. K Tsuchiya, S Katase, A Yoshino, and J Hachiya. Diffusion-weighted MR imaging of encephalitis. American Journal of Roentgenology 1999;173(4):10971099.

25. Kiroğlu Y, Calli C, Yunten N, Kitis O, Kocaman A, Karabulut N, Isaev H, Yagci B. Diffusion-weighted MR imaging of viral encephalitis. Neuroradiology. 2006;48(12):875-80.

26. Tsuchiya K, Hachiya J, Maehara T, Diffusion-weighted MR imaging in multiple sclerosis: comparison with contrast-enhanced study, Eur J Radiol. 1999;31(3):1659.

27. Chou MC, Lai PH, Yeh LR, Li JY, Yuan MK, Liang HL, Chen C, Pan HB, Lo YK, Yang CF. Posterior reversible encephalopathy syndrome: magnetic resonance imaging and diffusion-weighted imaging in 12 cases. Kaohsiung J Med Sci. 2004;20(8):381-8.

Source of Support: Nil; Conflict of Interest: None

Submitted: 27-03-2018; Published online: 30-03-2018 